\title{
The coupling effect of size and damage in micro-scale metallic materials
}

\author{
Haoxuan Ban ${ }^{a}$, Yin Yao ${ }^{b}$, Shaohua Chen ${ }^{\text {b, } c \text {, * }}$, Daining Fang b, c \\ a LNM, Institute of Mechanics, Chinese Academy of Sciences, Beijing, 100190, China \\ ${ }^{\mathrm{b}}$ Institute of Advanced Structure Technology and Beijing Key Laboratory of Lightweight Multi-functional Composite Materials and \\ Structures, Beijing Institute of Technology, Beijing, 100081, China \\ ${ }^{\mathrm{c}}$ Collaborative Innovation Center of Electric Vehicles in Beijing, Beijing Institute of Technology, Beijing, 100081, China
}

\section{A R T I C L E I N F O}

\section{Article history:}

Received 2 November 2016

Received in revised form 17 March 2017

Accepted 15 April 2017

Available online 5 May 2017

\section{Keywords:}

Micro-scale material

Strain gradient

Size effect

Damage effect

Intrinsic length scale

\begin{abstract}
A B S T R A C T
In order to characterize the deformation behavior accompanying damage of microstructures in micro-scale metallic materials, a new theoretical model is developed based on a low order strain gradient plasticity theory. Not only the size effect induced by strain gradient plasticity but also the one of microstructure damage induced by deformation is considered. The feature of the new theory includes two aspects: the strain gradient is taken as an internal variable to affect the tangential hardening modulus without the introduction of high-order stress or high-order boundary condition; both the elastic modulus and the involved intrinsic length are influenced by the microstructural damage. Two commonly used samples with size effect in micro-scales, i.e., the thin wire torsion and the ultra-thin beam bending, are re-analyzed with the new model. It is found that stiffness of the micro-scale material is gradually reduced along with the increasing deformation and the theoretical prediction is consistent well with the existing experimental data. All the results demonstrate that the present theory should be a promising way for predicting the mechanical behavior of a more complex system, for example, the micro-particle reinforced metal matrix composite and the recent-hot-studied nano-crystallized gradient materials.
\end{abstract} (c) 2017 Elsevier Ltd. All rights reserved.

\section{Introduction}

The miniaturization of electronic equipments and fabrication of advanced composites give rise to material systems with a

characteristic length on the order of micron or sub-micron meter. Within such a scale range, a strong size effect of mechanical behaviors has already been observed in many experiments. For examples, Fleck et al. (1994) found that in the micro-torsion test, the scaled torsional strength of a thin copper wire increases almost three times as the diameter of thin wires decreases from $170 \mu \mathrm{m}$ to $12 \mu \mathrm{m}$. Such size-dependent behavior was also observed in recent micro-torsion tests for polycrystalline copper and gold wires (Liu et al., 2013; Chen et al., 2015). Stolken and Evans (1998), Motz et al. (2008) and Zheng et al. (2016) reported a significant increase of beam bending strength with a decreasing thickness of ultra-thin nickel beams. Kiener et al. (2011) and Ryu et al. (2016) found that the yield strength and hardness of micropillars under compression and torsion

\footnotetext{
* Corresponding author. Institute of Advanced Structure Technology and Beijing Key Laboratory of Lightweight Multi-functional Composite Materials and Structures, Beijing Institute of Technology, Beijing, 100081, China.

E-mail addresses: chenshaohua72@hotmail.com, shchen@bit.edu.cn (S. Chen).
} 
increase with a deceasing diameter. Based on micro-forming tests of brass, Ran et al. (2013, Ran and Fu, 2014) verified that ductile fracture is difficult to occur when the grain size of metallic material is relatively small. For different micro-particle reinforced composites, Lloyd (1994), Sajjadi et al. (2011) and Liu et al. (2014) found an obvious improvement of flow strength when the volume fraction of particles keeps unchanged but with a decreasing diameter. More experimental evidences of size effect were provided by micro-indentation test, in which the material hardness was found to increase with a reducing indentation depth (for examples, Nix and Gao, 1998; Choi et al., 2012; Li et al., 2015; Zhao et al., 2016).

Unfortunately, the classical plasticity theory fails to characterize the size dependent phenomena since no internal length scale is involved in the constitutive relation. Based on the microscopic mechanism of plastic deformation and hardening (Cottrell, 1964; Ashby, 1970), strain gradient plasticity theories were developed, in which an intrinsic length scale of materials was introduced. Such a kind of theory was very popular to account for the size effect in micro-scale metallic materials.

The strain gradient plasticity theory can be generally divided into two classes. The first one involves high-order stresses as the work-conjugate to the strain gradient with high-order boundary conditions. Typical examples include Fleck et al. (1994), Fleck and Hutchinson (1997), Gao et al. (1999), Hwang et al. (2003), Yi et al. (2010) and Guha et al. (2013). Another framework retains the essential structure of the classical plasticity without any high-order stress, in which the strain gradient comes into play through the incremental plastic hardening modulus or a yield criterion. Relevant works include Acharya and Bassani (1995), Chen and Wang (2000, 2002a), Gao and Huang (2001), Abu Al-Rub and Voyiadjis (2006) and Askari et al. (2015).

With the strain gradient plasticity theories, size effect found in various micro-tests can be effectively predicted, such as the thin wire torsion and ultrathin beam bending (Fleck et al., 1994; Chen and Wang, 2000, 2002a; Huang et al., 2000; Gao and Huang, 2001; Mao et al., 2013; Bardella and Panteghini, 2015), the micro- and nano-indentation (Nix and Gao, 1998; Abu AlRub and Voyiadjis, 2006; Chen et al., 2007; Ouyang et al., 2010; Ma et al., 2012), compression of micropillars (Kiener et al., 2011; Zhang et al., 2014; Lin et al., 2016) as well as uniaxial compression and tension of particle-reinforced metal matrix composites (PMMC) (Fleck and Hutchinson, 1997; Chen and Wang, 2002b; Aghababaei and Joshi, 2011; Azizi et al., 2013; Legarth, 2015).

The strain gradient theories have also been successfully applied to study some other interesting issues at micro-scales. For instances, finite element method (FEM) combining strain gradient plasticity theories have been implemented to explain the cleavage fracture near the crack tip (Xia and Hutchinson, 1996; Chen and Wang, 2002c; Huang et al., 2014; Martinez-Paneda et al., 2016; Martinez-Paneda and Niordson, 2016). Strain gradient theories were also used to analyze void size effect on the stress distribution and void growth in porous solids (Liu et al., 2003; Wen et al., 2005; Monchiet and Bonnet, 2013). Furthermore, strain gradient constitutive models were established for crystalline solids in order to reveal the grain size effect on mechanical properties of single-, bi- and poly-crystals (Smyshlyaev and Fleck, 1996; Siddiq et al., 2007; Wu et al., 2012; Xiao et al., 2015; Lyu et al., 2015). Acharya and Beaudoin et al. proposed a grain-size dependent hardening law for viscoplastic poly-crystals, in which an intrinsic length scale was incorporated into the constitutive equation via a gradient measure of lattice incompatibility (Acharya and Beaudoin, 2000; Beaudoin et al., 2000). Tang et al. (2004, 2005) subsequently developed FEM models based on Acharya and Beaudoin's theory, which were applied to predict the crack tip fields in a single crystal and the directional dependence of crack growth along interfaces in a bi-crystal.

Though plenty of works have been carried out in the field of strain gradient plasticity, few attentions have been paid to the inservice damage caused by the material deformation. This issue has in fact been believed to be one of the main reasons resulting in the deviation between the experimental data and the strain gradient theoretical predictions (Chen and Wang, 2002b; Jiang and Tohgo, 2011; Martinez-Paneda and Betegon, 2015), especially for micro-particle reinforced composites. On the other hand, without considering the strain gradient effect, the classical continuum damage mechanics was found to be failure in predicting size-dependent degradation in micro-test of thin metal film and PMMC (Yang et al., 2008; Scudino et al., 2009; Liu et al., 2012). In view of these problems, a strain gradient theory considering the in-service damage effect simultaneously is needed.

Within the framework of nonlocal continuum theory (Eringen, 2002), a few of gradient-enhanced damage models were proposed for ductile materials, in which not only an intrinsic length parameter via a second-order gradient of plastic strain but also a strain-dependent damage variable is introduced in the constitutive relation (Voyiadjis and Dorgan, 2001; Engelen et al., 2003; Lele and Anand, 2009; Sciarra, 2012; Placidi, 2016). Such kind of model can eliminate the spurious meshdependency in numerical solutions, even though the high order terms in governing equations bring more cumbersomeness to the computational implementation. On the other hand, strain gradient plasticity theories were used to study the problem of shear band localization (another kind of material damage during the loading process, also called as failure) (Zbib and Aifantis, 1992; Shi et al., 2009; Chen et al., 2011; Zhu et al., 2016) and softening of a bar under a uniaxial tension (Engelen et al., 2006; Borg, 2007). Such a damage behavior was characterized by a negative softening modulus in these works, which is used to describe the softening stage after the strain hardening one. It is different from the in-service damage considered in the present paper, which happens along with the deformation, even in the elastic stage due to the microstructure evolution. However, determination of the intrinsic length parameter involved in the strain gradient plasticity theories is always a debatable problem, which, instead of a constant, should be related to the plastic deformation and microstructure characteristics (Nix and Gao, 1998; Abu Al-Rub and Voyiadjis, 2004; Voyiadjis and Abu Al-Rub, 2005; Faghihi and Voyiadjis, 2012). Recently, Liu and Elsayed (2015) also found that better agreement between theoretical predictions and experimental data for the thin wire torsion can be achieved with a decreasing intrinsic length, which they suggested may be attributed to the ductile 
damage inside the micro-scale materials. How to formulate the intrinsic length parameter as a function of material damage remains an open question.

The main aim of this paper is to establish a new framework of strain gradient theory with material damage taken into consideration. Based on the continuum damage mechanics (Lemaitre, 1992), a strain-dependent damage variable is defined, which is further introduced into the low-order strain gradient constitutive law proposed by Chen and Wang (2000) and the formula of intrinsic length parameter given by Nix and Gao (1998). Size effect is induced by the strain gradient, while damage effect is resulted from deformation. The key feature of the new theory is that both the instantaneous tangent modulus and the intrinsic length parameter will be influenced by the material damage parameter, while the strain gradient theory with microstructure damage evolution still retains the essential framework of the incremental version of conventional J2 deformation theory and obeys thermodynamic restrictions. The present constitutive relation considering both the size effect and the damage one describes the material behaviors in the elastic and elastic-plastic stages, which is used to predict size effect in thin wire torsion and ultrathin beam bending in this paper. Both results agree well with the experimental measurements.

\section{Brief review of the $C-W$ strain gradient theory}

Inspired by Acharya and Bassani (1995), in which they suggested that the strain gradient in a plastic flow theory could serve as an internal variable to increase the current tangential modulus, Chen and Wang (2000) proposed a low-order strain gradient theory retaining the essential structure of the incremental version of the conventional $J_{2}$ deformation theory, but with a new incremental hardening law,

$$
\dot{\sigma}_{e}=A^{\prime}\left(\varepsilon_{e}\right)\left(1+\frac{l^{2} \eta^{2}}{\varepsilon_{e}^{2}}\right)^{\alpha} \dot{\varepsilon}_{e}=B\left(\varepsilon_{e}, l \eta\right) \dot{\varepsilon}_{e}
$$

where the superposed dot denotes the material time derivative and $A^{\prime}\left(\varepsilon_{e}\right)$ denotes the current tangential modulus. $\alpha$ is an exponent. $\sigma_{e} \sqrt{3 \sigma_{i j}^{\prime} \sigma_{i j}^{\prime} / 2}$ is the Von Mises effective stress as a work conjugate of the effective strain $\varepsilon_{e}=\sqrt{2 \varepsilon_{i j}^{\prime} \varepsilon_{i j}^{\prime} / 3}$, with $\sigma_{i j}^{\prime}-\sigma_{i j}-\sigma_{m} \delta_{i j} / 3, \varepsilon_{i j}^{\prime}-\varepsilon_{i j}-\varepsilon_{m} \delta_{i j} / 3, \sigma_{m}=\sigma_{k k}, \varepsilon_{m}=\varepsilon_{k k} . \sigma_{i j}$ and $\varepsilon_{i j}$ represent the stress and strain tensors $(i, j=1,2,3)$, with the deviatoric stress and deviatoric strain $\sigma_{i j}^{\prime}$ and $\varepsilon_{i j}^{\prime}$, respectively. $\eta$ denotes the strain gradient term, which is defined as

$$
\eta=\sqrt{c_{1} \eta_{i j k}^{(1)} \eta_{i j k}^{(1)}+\chi_{e}^{2}}
$$

with

$$
l=l_{c S}, c_{1}=\left(\frac{l_{1}}{l_{c S}}\right)^{2}, \chi_{e} \sqrt{\frac{2}{3} \chi_{i j} \chi_{i j}}, \chi_{i j}=\theta_{i, j}, \theta=\frac{1}{2} e_{i j} u_{k, j}
$$

$\eta_{i j k}^{(1)}$ is the stretch gradient (Fleck and Hutchinson, 1997), $\chi_{e}$ is the effective rotation gradient. $l_{1}$ and $l_{c s}$ are intrinsic length scales for stretch and rotation gradients, respectively; $\chi_{i j}$ and $\theta_{i}$ represent the rotation gradient tensor and rotation vector, respectively;

The constitutive relation of the low-order strain gradient plasticity theory is in an incremental form (Chen and Wang, 2000).

$$
\dot{\sigma}_{i j}^{\prime}=\frac{2}{3} \frac{\varepsilon_{i j}^{\prime}}{\varepsilon_{e}} \dot{\sigma}_{e}+\frac{2}{3} \frac{\dot{\varepsilon}_{i j}^{\prime}}{\varepsilon_{e}} \sigma_{e}-\frac{2}{3} \frac{\varepsilon_{i j}^{\prime} \sigma_{e}}{\varepsilon_{e}^{2}} \dot{\varepsilon}_{e}
$$

It shows that the C-W theory involves neither high-order stress nor extra boundary conditions, which has successfully predicted size effect observed in several experiments, for examples, thin wire torsion, ultrathin beam bending, microindentation of pure metals and film-substrate system, as well as cleavage fracture at crack tip. However, the effect of micro-structural damage accompanying deformation can not be characterized by the original C-W theory.

\section{A strain gradient theory considering the effect of micro-structural damage}

\subsection{The damage variable}

The Cauchy stress $\sigma_{i j}$ satisfying the equilibrium equation $\boldsymbol{\sigma} \cdot \nabla+\boldsymbol{f}=0$ in a damaged material is different from the stress $\widehat{\sigma}_{i j}$ in an undamaged body (Lemaitre, 1992). The two stresses can be linked by a damage parameter $D$, 


$$
\sigma_{i j}=(1-D) \widehat{\sigma}_{i j}
$$

It shows that the introduced damage parameter characterizes a loss of load bearing capacity of the damaged material. In this paper, an isotropic damage case is considered so that $D$ is taken as a scalar (Lemaitre, 1992).

From a microscopic point of view, damage of a material is closely related to nucleation, coalescence and evolution of microcracks and voids, which are induced by microstructural deformation. Therefore, the damage parameter can be phenomenologically taken as a function of the effective strain $\varepsilon_{e}$. Assuming microstructural damage initiating with deformation, an empirical function has been proposed to describe the damage evolution (Engelen et al., 2003; Chen and Ghosh, 2012),

$$
D=\frac{\varepsilon_{e}}{\varepsilon_{e c}}
$$

where $\varepsilon_{e c}$ is defined as a critical effective strain for complete failure of materials. Therefore, the range of $D$ lies between 0 and 1 in the interval of $0 \leq \varepsilon_{e} \leq \varepsilon_{e c}$, which characterizes damage variation in the whole deformation process of a material.

\subsection{A damage-dependent intrinsic length scale}

The intrinsic length $l$ in strain gradient plasticity theories was always taken as a constant (Fleck et al., 1994; Gao et al., 1999; Chen and Wang, 2002a). However, many other studies suggested that the intrinsic length should change with the deformation of microstructure (Nix and Gao, 1998; Abu Al-Rub and Voyiadjis, 2004; Voyiadjis and Abu Al-Rub, 2005). A recent research further revealed that the intrinsic length of a material may be reduced by damage (Liu and Elsayed, 2015). A relationship between the damage variable and the intrinsic length scale should be established.

According to Nix and Gao (1998) and Gao and Huang (2001), the intrinsic length $l$ of a perfectly elasto-plastic material can be expressed as

$$
l=18 \lambda^{2}\left(\frac{\mu}{\sigma_{Y}}\right)^{2} b
$$

where $\lambda$ is an empirical constant usually on the order of $1 ; \mu$ is the elastic shear modulus and $\sigma_{Y}$ is the yield stress in the absence of strain gradient; $b$ is the Burgers vector.

As demonstrated by uniaxial-tension test for metallic materials (Lemaitre, 1995), damage can hardly affect the yield stress $\sigma_{Y}$ since the elastic deformation is very small, while the Young's modulus should decrease by a factor of $1-D$, i.e., $\bar{E}=E(1-D)$. It consequently leads to a damaged shear modulus $\bar{\mu}=\mu(1-D)$. As a result, the intrinsic length of a damaged material can be obtained as

$$
\bar{l}=18 \lambda^{2}\left(\frac{\bar{\mu}}{\sigma_{Y}}\right)^{2} b=(1-D)^{2} l
$$

Combining Eqs. (6) and (8) shows that the intrinsic length $\bar{l}$ for a damaged material decreases with increasing damage and increasing plastic deformation, which is consistent with the suggestion proposed by Abu Al-Rub and Voyiadjis (2004) and Liu and Elsayed (2015).

\subsection{Strain gradient constitutive relation with damage effect}

The incremental constitutive relation in Eq. (4) still holds for a damaged material with the characteristic length scale in microor sub-micrometer. Damage effect on the Cauchy stress demonstrated in Eq. (5) also holds for the corresponding deviatoric stress components, i.e.,

$$
\sigma_{i j}^{\prime}=(1-D) \widehat{\sigma}_{i j}^{\prime}
$$

where $\sigma_{i j}^{\prime}$ and $\widehat{\sigma}_{i j}^{\prime}$ denote the deviatoric stress components in the damaged and undamaged materials, respectively. Then, we have

$$
\sigma_{e}=(1-D) \widehat{\sigma}_{e}
$$


where $\sigma_{e}=\sqrt{3 \sigma_{i j}^{\prime} \sigma_{i j}^{\prime} / 2}$ is the effective stresses in the damaged material and $\sigma_{e}=\sqrt{3 \sigma_{i j}^{\prime} \sigma_{i j}^{\prime} / 2}$ is the one in the undamaged material.

Replacing the intrinsic length $l$ for an undamaged material with $\bar{l}$ for a damaged material in the incremental hardening law proposed by Chen and Wang (2000) for strain gradient plasticity, we can re-write Eq. (1) as

$$
\dot{\hat{\sigma}}_{e}=A^{\prime}\left(\varepsilon_{e}\right)\left(1+\frac{\bar{l}^{2} \eta^{2}}{\varepsilon_{e}^{2}}\right)^{a} \dot{\varepsilon}_{e}=B\left(\varepsilon_{e}, \bar{l} \eta\right) \dot{\varepsilon}_{e}
$$

where $\bar{l}$ abides by Eq. (8).

Integrating Eq. (11) yields

$$
\widehat{\sigma}_{e}=\int_{0}^{\varepsilon_{e}} A^{\prime}(\beta)\left(1+\frac{\bar{l}^{2} \eta^{2}}{\beta^{2}}\right)^{\alpha} d \beta
$$

where $\beta$ represents an integration variable ranging from 0 to $\varepsilon_{e}$.

The incremental form of Eq. (10) can be written as

$$
\dot{\sigma}_{e}=(1-D) \dot{\hat{\sigma}}_{e}-\dot{D} \widehat{\sigma}_{e}
$$

Substituting Eqs. (11) and (12) into Eq. (13) yields,

$$
\dot{\sigma}_{e}=C\left(\varepsilon_{e}, \bar{l} \eta\right) \dot{\varepsilon}_{e}
$$

where

$$
C\left(\varepsilon_{e} \bar{l} \eta\right)=\left[1-D\left(\varepsilon_{e}\right)\right] A^{\prime}\left(\varepsilon_{e}\right)\left(1+\frac{\bar{l}^{2} \eta^{2}}{\varepsilon_{e}^{2}}\right)^{\alpha}-\frac{d D}{d \varepsilon_{e}} \int_{0}^{\varepsilon_{e}} A^{\prime}(\beta)\left(1+\frac{\bar{l}^{2} \eta^{2}}{\varepsilon_{e}^{2}}\right)^{\alpha} d \beta
$$

Equation (15) denotes the incremental hardening relation for a damaged material with the characteristic length in micro- or sub-micro meters.

Comparing with the conventional J2 deformation theory, the present theory includes not only the effect of strain gradient plasticity but also the effect of microstructural damage. Furthermore, the microstructural damage evolution influences not only the instantaneous tangential hardening modulus but also the intrinsic length. The equilibrium equation still obeys the classical framework of the conventional plasticity theory without additional boundary conditions in contrast to high-order strain gradient plasticity theories (Fleck et al., 1994; Fleck and Hutchinson, 1997; Gao et al., 1999) and gradient-enhanced damage theories (Voyiadjis and Dorgan, 2001; Engelen et al., 2003; Sciarra, 2012).

\section{Comparison between theoretical predictions and experimental measurements}

Cases of thin wire torsion and ultrathin beam bending are analyzed with the above theory. Only the mechanical behavior in the hardening stage is studied since few experiments gave the measured results in the softening stage for the two structures.

\subsection{Thin wire torsion}

As shown in Fig. 1, a Cartesian coordinate system $\left(x_{1}, x_{2}, x_{3}\right)$ and a cylindrical polar coordinate system $\left(r, \theta, x_{3}\right)$ are attached to a thin wire with the longitudinal axis in the $x_{3}$ direction. The center of the circular cross-section of the wire coincides with the origin of $\left(x_{1}, x_{2}\right)$ or $(r, \theta)$ plane. The radius of the wire is $a . \kappa$ is the twist per unit length of the wire and taken to be positive without loss of generality. The displacement field can be written as

$$
u_{1}=-\kappa x_{2} x_{3}, \quad u_{2}=\kappa x_{1} x_{3}, \quad u_{3}=0
$$

which yield the torsion-induced shear strains in the Cartesian coordinate system 


$$
\varepsilon_{13}=\varepsilon_{31}=-\frac{1}{2} \kappa x_{2}, \quad \varepsilon_{23}=\varepsilon_{32}=\frac{1}{2} \kappa x_{1}
$$

and the non-vanishing components of the rotation gradient tensor.

$$
\chi_{11}=\chi_{22}=-\frac{1}{2} \kappa, \quad \chi_{33}=\kappa
$$

Then the effective strain and the effective rotation gradient can be obtained as,

$$
\varepsilon_{e}=\frac{1}{\sqrt{3}} \kappa r, \quad \chi_{e}=\kappa
$$

For a thin wire under pure torsion, the stretch gradient in Eq. (2) equals zero according to Smyshlyaev and Fleck (1996),

$$
\eta_{i j k}^{(1)} \eta_{i j k}^{(1)}=0
$$

Combining Eqs. (2) (19) and (20) leads to $\eta=\kappa$.

According to Fleck et al. (1994) and Chen and Wang (2000), a simple power law is adopted to describe the constitutive relationship between the effective stress $\sigma_{e}$ and effective strain $\varepsilon_{e}$ for a perfectly elasto-plastic thin wire,

$$
\sigma_{e}=A\left(\varepsilon_{e}\right)=\sigma_{0} \varepsilon_{e}^{N}
$$

where $\sigma_{0}$ and $N$ are material constants.

Then the relation for a damaged elasto-plastic thin wire can be obtained according to Eq. (14) as

$$
\dot{\sigma}_{e}=f\left(\varepsilon_{e}\right) \dot{\varepsilon}_{e}
$$

where

$$
f\left(\varepsilon_{e}\right)=\left[1-D\left(\varepsilon_{e}\right)\right] N \sigma_{0} \varepsilon_{e}^{N-1}\left\{1+\frac{3\left[1-D\left(\varepsilon_{e}\right)\right] l^{2}}{r^{2}}\right\}^{\alpha}-\frac{d D}{d \varepsilon_{e}} \int_{0}^{\varepsilon_{e}} N \sigma_{0} \beta^{N-1}\left\{1+\frac{3[1-D(\beta)] l^{2}}{r^{2}}\right\}^{\alpha} d \beta
$$

Integrating Eq. (22) results in $\sigma_{e}$. The shear stresses in the wire under pure torsion are

$$
\tau_{13}=\tau_{31}=\frac{2 \varepsilon_{13}}{3 \varepsilon_{e}} \sigma_{e}, \quad \tau_{23}=\tau_{32}=\frac{2 \varepsilon_{23}}{3 \varepsilon_{e}} \sigma_{e}
$$

which can be further expressed in the cylindrical coordinate system as
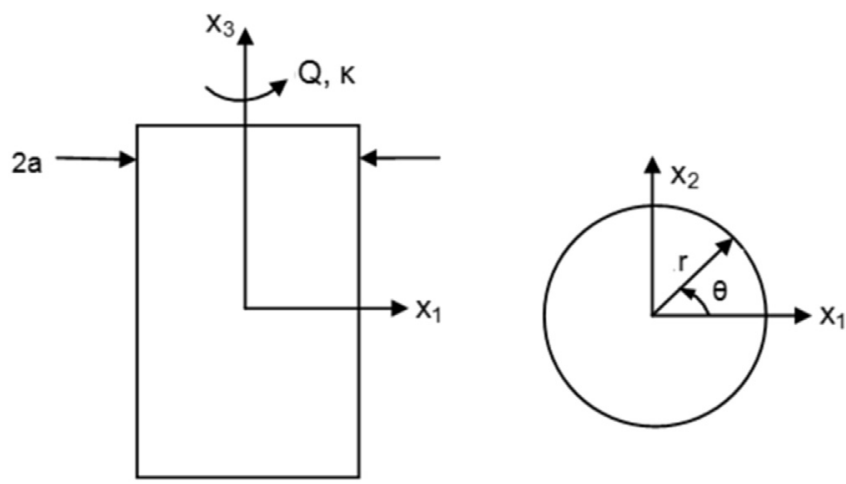

Fig. 1. Schematic of a thin wire under torsion. Both a Cartesian coordinate system $\left(x_{1}, x_{2}, x_{3}\right)$ and a polar one $\left(r, \theta, x_{3}\right)$ are attached to the thin wire of radius $a$. $Q$ is the torque and $\kappa$ is the twist per unit length of the wire. 


$$
\tau_{\theta z}=\frac{1}{\sqrt{3}} \sigma_{e}=\frac{1}{\sqrt{3} \int_{0}^{\varepsilon_{e}} f(\varsigma) d \varsigma}
$$

The overall torque induced by $\tau_{\theta z}$ can be obtained,

$$
Q=\int_{0}^{2 \pi} \int_{0}^{a} \tau_{\theta z} r^{2} d r d \theta=\frac{1}{\sqrt{3}} \int_{0}^{2 \pi} \int_{0}^{a} \tau_{\theta z} r^{2} d r d \theta=\frac{1}{\sqrt{3}} \int_{0}^{2 \pi} \int_{0}^{a} r^{2} \int_{0}^{\varepsilon_{e}} f(\varsigma) d \varsigma d r d \theta
$$

based on which the relation between the overall torque and surface strain $\varepsilon_{w}=\kappa a$ can be established. Detailed analysis is given in Section 5 .

\subsection{Ultrathin beam bending}

Fig. 2 shows an ultrathin beam with its longitudinal axis in the $x_{1}$ direction. Bending occurs in the $\left(x_{1}, x_{2}\right)$ plane. The model can be regarded as a plane stress plate in the $x_{2}$ direction due to the very thin thickness and a plane strain case in the out of plane $\left(x_{3}\right)$ direction. $k$ denotes the bending curvature. $h$ and $b$ are the beam's thickness and width, respectively. Due to the small deformation in bending, the displacement field is

$$
u_{1}=\kappa x_{1} x_{2}, \quad u_{2}=-\kappa\left(x_{1}^{2}+x_{2}^{2}\right) / 2, \quad u_{3}=0
$$

which lead to the non-zero strain components

$$
\varepsilon_{11}=-\varepsilon_{22}=\kappa x_{2}
$$

and the non-zero component of rotation gradient tensor.

$$
\chi_{31}=-\kappa
$$

Using Eqs. (28) and (29) leads to the effective strain and the effective rotation gradient,

$$
\varepsilon_{e}=\frac{2}{\sqrt{3}} \kappa\left|x_{2}\right|, \chi_{e}=\sqrt{\frac{2}{3}} \kappa
$$

The stretch gradient is (Chen and Wang, 2000)

$$
\eta_{i j k}^{(1)} \eta_{i j k}^{(1)}=\frac{76}{75} \kappa^{2}
$$

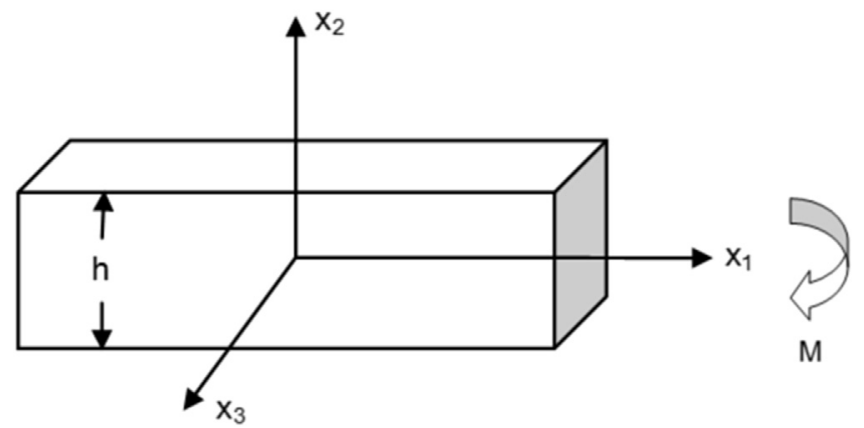

Fig. 2. Schematic of a thin beam under bending, where $\left(x_{1}, x_{2}, x_{3}\right)$ is a Cartesian coordinate system, $h$ is the beam thickness and $M$ is the bending moment. 
According to Smyshlyaev and Fleck (1996), $l_{c s}$ is much larger than $l_{1}$, which results in a very small $c_{1}$ in Eq. (2). Considering the same order of magnitude of the stretch gradient $\eta_{i j k}^{(1)} \eta_{i j k}^{(1)}$ as the effective curvature $\chi_{e}^{2}$, we can omit the term of $\eta_{i j k}^{(1)} \eta_{i j k}^{(1)}$ for simplicity, which leads to $\eta=\chi_{e}$. That is only the rotation gradient is considered for the beam bending behavior.

According to the tensile test of thin foil-beams (Stolken and Evans, 1998), a piecewise linear function was adopted to describe the constitutive relationship between the effective stress and effective strain (Chen and Wang, 2000),

$$
\left\{\begin{array}{cc}
\sigma_{e}=A\left(\varepsilon_{e}\right)=\widehat{\Sigma}_{0}+\widehat{E}_{p} \varepsilon_{e} & \varepsilon \geq \varepsilon_{0} \\
\sigma_{e}=A\left(\varepsilon_{e}\right)=E_{e} & \varepsilon \leq \varepsilon_{0}
\end{array}\right.
$$

where $\varepsilon_{0}=\sqrt{3} \Sigma_{0} /(2 E)$ is the yield strain, and $\widehat{\Sigma}_{0} 2 \sqrt{3} \Sigma_{0} E /\left(4 E+3 E_{p}\right), \widehat{E}_{p}=3 E_{p} E /\left(4 E+3 E_{p}\right)$. Here, $\Sigma_{0}$ is the yield strength, $E$ and $E_{p}$ represent the Young's modulus and linear hardening modulus, respectively.

Substituting Eq. (32) into Eq. (15) leads to an incremental relation considering both effects of strain gradient and damage,

$$
\left\{\begin{array}{c}
\dot{\sigma}_{e}\left[1-D\left(\varepsilon_{e}\right)\right] \widehat{E}_{p}\left(1+\frac{\bar{l}^{2} \eta^{2}}{\varepsilon_{e}^{2}}\right)^{\alpha} \dot{\varepsilon}_{e}-\frac{d D}{d \varepsilon_{e}} \dot{\varepsilon}_{e} \int_{0}^{\varepsilon_{e}} \widehat{E}_{p}\left(1+\frac{\bar{l}^{2} \eta^{2}}{\beta^{2}}\right)^{\alpha} d \beta \quad \varepsilon_{e} \geq \varepsilon_{0} \\
\dot{\sigma}_{e}\left[1-D\left(\varepsilon_{e}\right)\right] E\left(1+\frac{\bar{l}^{2} \eta^{2}}{\varepsilon_{e}^{2}}\right)^{\alpha} \dot{\varepsilon}_{e}-\frac{d D}{d \varepsilon_{e}} \dot{\varepsilon}_{e} \int_{0}^{\varepsilon_{e}} E_{p}\left(1+\frac{\bar{l}^{2} \eta^{2}}{\beta^{2}}\right)^{\alpha} d \beta \quad \varepsilon_{e} \geq \varepsilon_{0}
\end{array}\right.
$$

Substituting Eq. (30) into Eq. (33) yields.

$$
\left\{\begin{array}{c}
\dot{\sigma}_{e}=\left[1-D\left(\varepsilon_{e}\right)\right]\left\{1+\left[1-D\left(\varepsilon_{e}\right)\right]^{4} \frac{l^{2}}{2 x_{2}^{2}}\right\}^{\alpha} \widehat{E}_{p} \dot{\varepsilon}_{e}-\frac{d D}{d \varepsilon_{e}} \dot{\varepsilon}_{e} \int_{0}^{\varepsilon_{e}} \widehat{E}_{p}\left\{1+[1-D(\beta)]^{4} \frac{l^{2}}{2 x_{2}^{2}}\right\}^{\alpha} d \beta \quad \varepsilon_{e} \geq \varepsilon_{0} \\
\dot{\sigma}_{e}=\left[1-D\left(\varepsilon_{e}\right)\right]\left\{1+\left[1-D\left(\varepsilon_{e}\right)\right]^{4} \frac{l^{2}}{2 x_{2}^{2}}\right\}^{\alpha} E \dot{\varepsilon}_{e}-\frac{d D}{d \varepsilon_{e}} \dot{\varepsilon}_{e} \int_{0}^{\varepsilon_{e}} E_{p}\left\{1+[1-D(\beta)]^{4} \frac{l^{2}}{2 x_{2}^{2}}\right\}^{\alpha} d \beta \quad \varepsilon_{e} \leq \varepsilon_{0}
\end{array}\right.
$$

Integrating Eq. (34) can find $\sigma_{e}$.

Substituting Eqs. (28) and (30) into the deviatoric stress component $\sigma_{i j}^{\prime}=\frac{2 \sigma_{e}}{3 \varepsilon_{e}} \varepsilon_{i j}$ yields.

$$
\sigma_{11}^{\prime}=-\sigma_{22}^{\prime}=\frac{\operatorname{sign}\left(x_{2}\right)}{\sqrt{3}} \sigma_{e}
$$

Considering the plane stress condition in the thickness direction and plane strain condition in the width direction leads to

$$
\sigma_{11}=\frac{2 \operatorname{sign}\left(x_{2}\right)}{\sqrt{3}} \sigma_{e}, \quad \sigma_{33}=\frac{2 \operatorname{sign}\left(x_{2}\right)}{\sqrt{3}} \sigma_{e}
$$

The moment acted on the thin beam can then be obtained as

$$
M=2 \int_{0}^{h / 2} \sigma_{11} b x_{2} d x_{2}
$$

based on which the relation between the bending moment and surface strain $\varepsilon_{b}=\kappa h / 2$ is established.

\section{Results and discussion}

\subsection{Thin wire torsion}

The coupling effect of size and damage in thin copper wire torsion is analyzed based on Eq. (26). According to Fleck et al. (1994), the power exponent $N$ in the hardening law is taken as 0.22 and the intrinsic length scale $l$ in Eq. (8) is taken as $2.82 \mu \mathrm{m}$ for an undamaged copper wire (Chen and Wang, 2000). The critical effective strain $\varepsilon_{e c}$ is taken as 30 corresponding to $D=1$, which has the same order of magnitude as the fracture strain of metal wires (Dieter, 1986). 
Fig. 3 presents the torque versus surface strain for thin copper wires with diameters ranging from $12 \mu \mathrm{m}$ to $170 \mu \mathrm{m}$, in which the damage parameter abides by Eq. (6). Both the result predicted by C-W theory without damage effect and the experimentally measured data in Fleck et al. (1994) are given for comparison. It is found that both the theoretical predictions with and without damage effect agree well with the experimental data, while the former is slightly smaller than the latter for a thin wire with a fixed diameter and a given surface strain due to a reduction of the load bearing capacity resulted from damage. The small deviation between the two kinds of theoretical predictions further demonstrates that microstructural damage of the analyzed thin copper wire under torque is not very obvious in the involved strain range. It agrees with the fact that the purity of the copper used in Fleck et al. (1994) is much high, leading to internal weak damage during the deformation process.

\subsection{Ultrathin beam bending}

Experimental test on the bending moment of ultrathin nickel beams with different thickness has been carried out by Stolken and Evans (1998), in which the bending moment is influenced significantly by the beam thickness. The intrinsic length scale for nickel beams was predicted to be $l=3.3 \mu \mathrm{m}$ by the C-W strain gradient plasticity theory without damage effect (Chen and Wang, 2000). The yield strength and hardening modulus for beams with different thickness are given in Table 1 according to the tensile test of nickel foils (Stolken and Evans, 1998). Considering the microstructural damage effect during the beam bending process, we analyze the bending moment based on Eq. (37), in which not only the size effect but also the damage one is included. The damage function given in Eq. (6) is adopted, in which the critical effective strain $\varepsilon_{\text {ec }}$ is taken to be 0.5 without loss of generality for an ultrathin bending beam.

The predicted bending moment as a function of the surface strain for nickel beams with different widths is shown in Fig. 4 . The experimental data as well as the result predicted by the C-W strain gradient plasticity theory without damage effect is also given for comparison. It is found that both the theoretical predictions with and without microstructural damage effect agree well with the experimental results. In contrast, the former one agree better with the experimental measurements than the latter, which demonstrates that the microstructural damage is a little bit obvious in the beam bending experiment. Another interesting finding in Fig. 4 is that the thinner the beam, the more significant the damage effect would become. It suggests that a thicker beam with a larger bending rigidity exhibits a better resistance to the damage evolution.

\subsection{Comparison between the present theory and the Aifantis's theory}

Aifantis and Zbib et al. proposed a kind of gradient-dependent theory of plasticity (Aifantis, 1984, 1992; Zbib and Aifantis, 1992; Taylor et al., 2002), in which the yield function was formulated as

$$
F=\tau-k\left(\bar{\gamma}, \nabla^{2} \bar{\gamma}, \nabla \bar{\gamma}\right)=\tau-\left[k_{0}(\bar{\gamma})-c_{1} \nabla^{2} \bar{\gamma}-c_{2} \nabla \bar{\gamma} \cdot \bar{\gamma}\right]
$$

where $\tau$ is an effective stress (e.g., a von-Mises stress), $\bar{\gamma}$ is the accumulated effective plastic strain, $k\left(\bar{\gamma}, \nabla^{2} \bar{\gamma}, \nabla \bar{\gamma}\right)$ is a flow stress related to its classical counterpart $k_{0}(\bar{\gamma})$ (linear or exponential function of $\bar{\gamma}$ ) and the strain gradient terms, $c_{1}$ and $c_{2}$ are two coefficients associated with the intrinsic length scale. The tangent modulus of a material can be expressed as

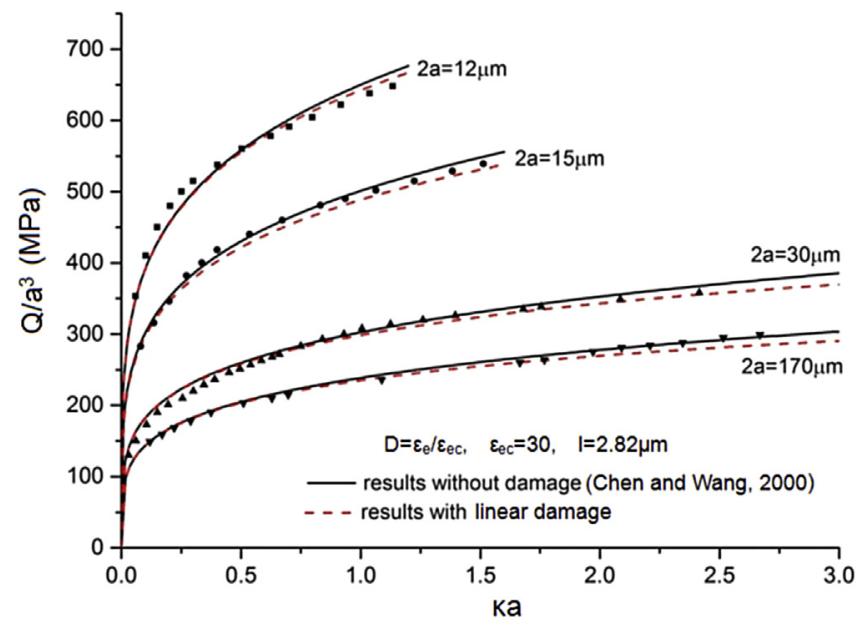

Fig. 3. Torque as a function of the surface strain for copper wires with different micro-meter diameters, in which the solid lines denote the C-W theoretical predictions without damage effect (Chen and Wang, 2000), the dashed lines denote the theoretical results predicted by the present strain gradient theory with damage effect, and the scattered symbols denote the experiment data given by Fleck et al. (1994). 
Table 1

Material parameters of nickel foils with different thickness (Stolken and Evans, 1998).

\begin{tabular}{lll}
\hline Foil thickness $(\mu \mathrm{m})$ & $\Sigma_{0}(\mathrm{MPa})$ & $\mathrm{E}_{\mathrm{p}}(\mathrm{GPa})$ \\
\hline 12.5 & 56 & 1.15 \\
25 & 75 & 1.30 \\
50 & 103 & 1.05 \\
\hline
\end{tabular}

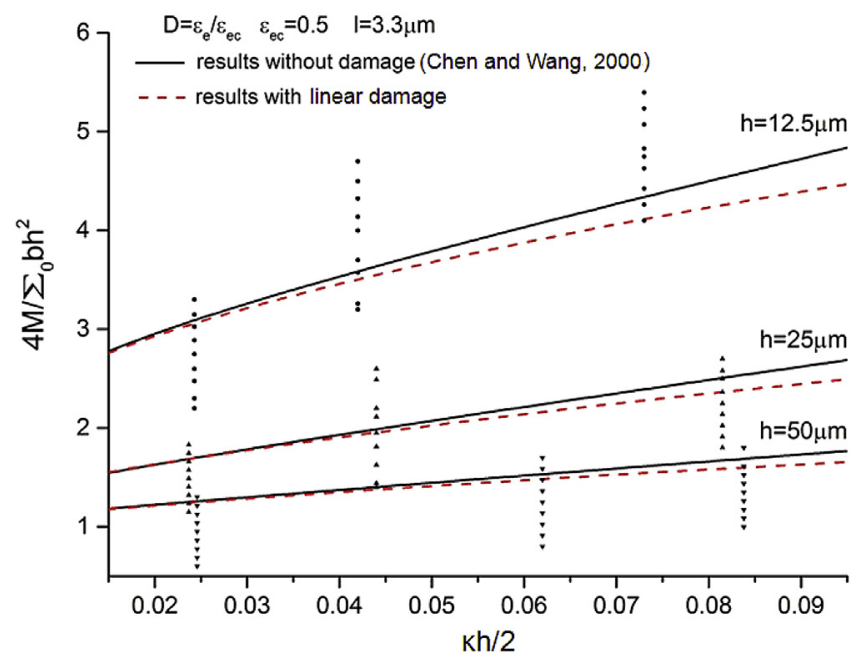

Fig. 4. Non-dimensional bending moment versus the surface strain for ultrathin nickel beams with different thickness, where the solid lines denote the C-W theoretical predictions without damage effect (Chen and Wang, 2000), the dashed lines denote the theoretical results predicted by the present strain gradient theory with damage effect, and the scattered symbols denote the experiment data given by Stolken and Evans (1998).

$$
\begin{gathered}
h=\frac{d k}{d \bar{\gamma}}=h_{0}-c_{1}^{\prime} \nabla^{2} \bar{\gamma}-c_{2}^{\prime} \nabla \bar{\gamma} \cdot \nabla \bar{\gamma} \\
h_{0}=\frac{d k_{0}}{d \bar{\gamma}}, c_{1}^{\prime}=\frac{d c_{1}}{d \bar{\gamma}}, c_{2}^{\prime}=\frac{d c_{2}}{d \bar{\gamma}}
\end{gathered}
$$

The yield function in such a strain gradient theory can be regarded as a hardening law for a positive tangential modulus $h$, and a softening one for a negative tangential modulus $h$. Usually, the mechanical behavior of most materials can be divided into three stages, the elastic deformation, the elastic-plastic deformation and the last softening stage. Sometimes, the softening region is also called as a damage stage, during which shear bands would happen. Strain gradient theories can also be well used to explain the behavior of shear bands when the tangential modulus takes a negative value at the softening stage, for examples, Aifantis (1992), Zbib and Aifantis (1992), Taylor et al. (2002), Chen et al. (2011).

In addition, Bammann and Aifantis proposed a physically-based gradient theory of continuum damage, in which the volume fraction of voids (porosity) was used as a measure of damage (Bammann and Aifantis, 1989; Solanki and Bammann, 2010). The intrinsic length scale of material was introduced into the elasto-plastic constitutive equations via a second-order gradient of porosity, so that the coupling effect of size and damage was considered. However, the porosity is not easy to determine in experiments (Yu and Feng, 1997).

Different from the strain gradient theories proposed by Aifantis and Zbib et al. (Aifantis, 1984, 1992; Zbib and Aifantis, 1992; Taylor et al., 2002). and the gradient theory of continuum damage (Bammann and Aifantis, 1989; Solanki and Bammann, 2010), the present theory has several unique features. (i) It belongs to a low-order strain gradient theory without any high-order gradient terms due to the introduction of incremental constitutive relation. The strain gradient can be looked as an internal variable. (ii) The damage parameter as a function of the effective plastic strain is introduced into the strain gradient hardening law to characterize the damage effect during the whole deformation process, which depends on the effective strain and the finally failure one as shown in Eq. (6). Such a definition of damage has been well adopted in the continuum damage mechanics due to the measurable parameters and consistence with experimental results. (iii) Since the intrinsic length depends on the material properties, it should depend on the damage parameter. An analytical relation between the intrinsic length and the damage parameter is established in the present model, in contrast to a constant one in the existing strain gradient theories. The instantaneous tangential modulus during deformation is also affected by the damage parameter due to 
the microstructure evolution. (iv) The present model can also be used to analyze the shear band problem only if the constitutive relation of a material includes the softening stage with a negative shear modulus.

\section{Conclusions}

A strain gradient plasticity theory considering the damage effect is developed, which remains not only the main feature of the low-order strain gradient theory proposed by Chen and Wang (2000) but also that of the continuum damage mechanics (Lemaitre, 1992). As a result, no high-order stress or high-order boundary condition is involved. Not only the tangential hardening modulus but also the intrinsic length involved in the strain gradient plasticity theory depends on the damage parameter, both of which are reduced by the microstructural damage. The proposed theory is further used to analyze the mechanical behaviors of thin wire torsion and ultrathin beam bending. Both the size effect and the damage-induced degradation of material bearing capacity are predicted well, though the damage effect is not very obvious in the two chosen tests. It is no doubt that the present theory should be very useful for understanding the decreasing hardening modulus found in micro-particle reinforced metal matrix composites (Lloyd, 1994; Liu et al., 2012; 2014) as well as the mechanical property of nano-crystalline gradient materials under external loading (Lu et al., 2000, 2009; Fang et al., 2011). Further study on the coupling effect of size and microstructural damage evolution in composites is forthcoming as well as the prediction of shear band width in metals.

\section{Acknowledgments}

The work reported here is supported by NSFC through Grants \#11372317, \#11532013, \#11402270, and the BIT Creative Research Plan.

\section{References}

Abu Al-Rub, R.K., Voyiadjis, G.Z., 2004. Analytical and experimental determination of the material intrinsic length scale of strain gradient plasticity theory from micro- and nano-indentation experiments. Int. J. Plast. 20 (6), 1139-1182.

Abu Al-Rub, R.K., Voyiadjis, G.Z., 2006. A physically based gradient plasticity theory. Int. J. Plast. 22 (4), 654-684.

Acharya, A., Bassani, J.L., 1995. On Non-local Flow Theories that Preserve the Classical Structure of Incremental Boundary Value Problems (In: Micromechanics of Plasticity and Damage of Multiphase Materials, IUTAM Symposium, Paris).

Acharya, A., Beaudoin, A.J., 2000. Grain-size effect in FCC viscoplasticpolycrystals at moderate strains. J. Mech. Phys. Solids 48 (10), $2213-2230$.

Aghababaei, R., Joshi, S.P., 2011. Grain size-inclusion size interaction in metal matrix composites using mechanism-based gradient crystal plasticity, 48 (18), $2585-2594$.

Aifantis, E.C., 1984. On the microstructural origin of certain inelastic models. ASME J. Eng. Mater. Technol. 106 (4), 326-330.

Aifantis, E.C., 1992. On the role of gradients in the localization of deformation and fracture. Int. J. Eng. Sci. 30 (10), 1279-1299.

Ashby, M.F., 1970. The deformation of plastically non-homogeneous alloys. Phil. Mag. 21 (170), 399-424.

Askari, H., Maughan, M.R., Abdolrahim, N., Sagapuram, D., Bahr, D.F., Zbib, H.M., 2015. A stochastic crystal plasticity framework for deformation of microscale polycrystalline materials. Int. J. Plast. 68, 21-33.

Azizi, R., Legarth, B.N., Niordson, C.F., 2013. A new macroscopically anisotropic pressure dependent yield function for metal matrix composite based on strain gradient plasticity for the microstructure. J. Mech. Phys. Solids 61 (4), 991-1009.

Bammann, D.J. Aifantis, E.C., 1989. A damage model for ductile metals. Nucl. Eng. Des. 116 (3), 355-362.

Bardella, L., Panteghini, A., 2015. Modelling the torsion of thin metal wires by distortion gradient plasticity. J. Mech. Phys. Solids 78, 467-492.

Beaudoin, A.J., Acharya, A., Chen, S.R., Korzekwa, D.A., Stout, M.G., 2000. Consideration of grain-size effect and kinetics in the plastic deformation of metal polycrystals. Acta Mater. 48 (13), 3409-3423.

Borg, U., 2007. Strain gradient crystal plasticity effects on flow localization. Int. J. Plast. 23 (8), 1400-1416.

Chen, S.H., Wang, T.C., 2000. A new hardening law for strain gradient plasticity. Acta Mater. 48 (16), 3997-4005.

Chen, S.H., Wang, T.C., 2002a. A new deformation theory for strain gradient effects. Int. J. Plast. 18 (8), 971-995.

Chen, S.H., Wang, T.C., 2002b. Size effects in the particle-reinforced metal-matrix composites. Acta Mech. 157 (1), $113-127$.

Chen, S.H., Wang, T.C., 2002c. Finite element solutions for plane strain mode I crack with strain gradient effects. Int. J. Solids Struct. 39 (5), $1241-1257$.

Chen, S.H., Liu, L., Wang, T.C., 2007. Small scale, grain size and substrate effects in nano-indentation experiment of film-substrate systems. Int. J. Solids Struct. 44 (13), 4492-4504.

Chen, S.H., Feng, B., Wei, Y.G., Wang, T.C., 2011. Prediction of the initial thickness of shear band localization based on a reduced strain gradient theory. Int. J. Solids Struct. 48 (21), 3099-3111.

Chen, Y.L., Ghosh, S., 2012. Micromechanical analysis of strain rate-dependent deformation and failure in composite microstructures under dynamic loading conditions. Int. J. Plast. 32-33, 218-247.

Chen, Y., Kraft, O., Walter, M., 2015. Size effects in thin coarse-grained gold microwires under tensile and torsional loading. Acta Mater. 87, 78-85.

Choi, I.C., Zhao, Y.K., Kim, Y.J., Yoo, B.G., Suh, J.Y., Ramamurty, U., Jang, J.I., 2012. Indentation size effect and shear transformation zone size in a bulk metallic glass in two different structural states. Acta Mater. 60 (19), 6862-6868.

Cottrell, A.H., 1964. The Mechanical Properties of Matter. Wiley, New York, p. 277.

Dieter, G.E., 1986. Mechanica Metallurgy, third ed. McGraw-Hill Book Company, Inc., New York.

Eringen, A.C., 2002. Nonlocal Continuum Field Theories. Springer, New York.

Engelen, R.A.B., Geers, M.G.D., Baaijens, F.P.T., 2003. Nonlocal implicit gradient-enhanced elasto-plasticity for the modeling of softening behavior. Int. J. Plast. $19(4), 403-433$

Engelen, R.A.B., Fleck, N.A., Peelings, R.H.J., Geers, M.G.D., 2006. An evaluation of higher-order plasticity theories for predicting size effects and localization. Int. J. Solids Struct. $43(7-8), 1857-1877$.

Faghihi, D., Voyiadjis, G.Z., 2012. Determination of nanoindentation size effects and variable material intrinsic length scale for body-centered cubic metals. Mech. Mater. 44, 189-211.

Fang, T.H., Li, W.L., Tao, N.R., Lu, K., 2011. Revealing extraordinary intrinsic tensile plasticity in gradient nano-grained copper. Science 331 (6024), 1587-1590.

Fleck, N.A., Muller, G.M., Ashby, M.F., Hutchinson, J.W., 1994. Strain gradient plasticity: theory and experiment. Acta Metall. Mater. 42 (2), $475-487$.

Fleck, N.A., Hutchinson, J.W., 1997. Strain gradient plasticity. In: Hutchinson, J.W., Wu, T.Y. (Eds.), Advances in Applied Mechanics, vol. 33. Academic Press, New York, pp. 295-361.

Gao, H.J., Huang, Y.G., Nix, W.D., Hutchinson, J.W., 1999. Mechanism-based strain gradient plasticity- I. Theory. J. Mech. Phys. Solids 47 (6), $1239-1263$. 
Gao, H.J., Huang, Y.G., 2001. Taylor-based nonlocal theory of plasticity. Int. J. Solids Struct. 38 (15), $2615-2637$.

Guha, S., Sangal, S., Basu, S., 2013. Finite Element studies on indentation size effect using a higher order strain gradient theory. Int. J. Solids Struct. 50 (6), $863-875$.

Huang, Y.G., Gao, H.J., Nix, W.D., Hutchinson, J.W., 2000. Mechanism-based strain gradient plasticity- II. Analysis. J. Mech. Phys. Solids 48 (1), 99-128.

Huang, M.S., Tong, J., Li, Z.H., 2014. A study of fatigue crack tip characteristics using discrete dislocation dynamics. Int. J. Plast. 54, $229-246$.

Hwang, K.C., Jiang, H., Huang, Y.G., Gao, H.J., 2003. Finite deformation analysis of mechanism-based strain gradient plasticity: torsion and crack tip field. Int. J. Plast. 19 (2), 235-251.

Jiang, Y.P., Tohgo, K., 2011. An incremental damage theory for micropolar composites taking account of progressive debonding and particle size effect. Comput. Mater. Sci. 50 (12), 3358-3364.

Kiener, D., Guruprasad, P.J., Keralavarma, S.M., Dehm, G., Benzerga, A.A., 2011. Work hardening in micropillar compression: In situ experiments and modeling. Acta Mater. 59 (10), 3825-3840.

Legarth, B.N., 2015. Plasticity dependent damage evolution in composites with strain-gradient effects. Int. J. Solids Struct. 63, 1-10.

Lele, S.P., Anand, L., 2009. A large-deformation strain-gradient theory for isotropic viscoplastic materials. Int. J. Plast. 25 (3), $425-453$.

Lemaitre, J., 1992. A Course on Damage Mechanics. Springer, Berlin.

Lemaitre, J., 1995. A continuous damage mechanics model for ductile fracture. ASME J. Eng. Mater. Technol. 107 (1), $83-89$.

Li, J.H., Li, F.G., Ma, X.K., Wang, Q.R., Dong, J.Z., Yuan, Z.W., 2015. A strain-dependent ductile damage model and its application in the derivation of fracture toughness by micro-indentation. Mater. Des. 67, 623-630.

Lin, P., Liu, Z.L., Zhuang, Z., 2016. Numerical study of the size-dependent deformation morphology in micropillar compressions by a dislocation-based crystal plasticity model. Int. J. Plast. 87, 32-47.

Liu, B., Qiu, X., Huang, Y., Huang, K.C., Li, M., Liu, C., 2003. The size effect on void growth in ductile materials. J. Mech. Phys. Solids 51 (7), $1171-1187$.

Liu, B., Huang, W.M., Huang, L., Wang, H.W., 2012. Size-dependent compression deformation behaviors of high particle content B ${ }_{4}$ /Al composites. Mater. Sci. Eng. A 534 (1), 530-535.

Liu, B., Huang, W.M., Wang, H.W., Wang, M.L., Li, X.F., 2014. Study on the load partition behaviors of high particle content B ${ }_{4}$ /Al composites in compression. J. Compos. Mater. 48 (3), 355-364.

Liu, D.B., He, Y.M., Dunstan, D.J., Zhang, B., Gan, Z.P., Hu, P., Ding, H.M., 2013. Toward a further understanding of size effects in the torsion of thin metal wires: an experimental and theoretical assessment. Int. J. Plast. 41, 30-52.

Liu, J.X., Elsayed, T., 2015. A strain gradient plasticity theory with application to wire torsion. Int. J. Damage Mech. 24 (4), $512-528$.

Lloyd, D.J., 1994. Particle reinforced aluminium and magnesiummatrix composites. Inter. Mater. Rev. 39 (1), 1-23.

Lu, L., Sui, M.L., Lu, K., 2000. Superplastic extensibility of nanocrystalline copper at room temperature. Science 287 (5457), $1463-1466$.

Lu, K., Lu, L., Suresh, S., 2009. Strengthening materials by engineering coherent internal boundaries at the nanoscale. Science 324 (5925), $349-352$.

Lyu, H., Taheri-Nassaj, N., Zbib, H.M., 2015. A multiscale gradient-dependent plasticity model for size effects. Philo. Mag. 96 (18), $1883-1908$.

Ma, Z.S., Zhou, Y.C., Long, S.G., Lu, C., 2012. On the intrinsic hardness of a metallic film/substrate system: indentation size and substrate effects. Int. J. Plast. $34,1-11$.

Mao, Y.Q., Ai, S.G., Fang, D.N., Fu, Y.M., Chen, C.P., 2013. Elasto-plastic analysis of micro FGM beam basing on mechanism-based strain gradient plasticity theory. Compos. Struct. 101, 168-179.

Martinez-Paneda, E., Betegon, C., 2015. Modeling damage and fracture within strain-gradient plasticity. Int. J. Solid Struct. 59, 208-215.

Martinez-Paneda, E., Niordson, C.F., Gangloff, R.P., 2016. Strain gradient plasticity-based modeling of hydrogen environment assisted cracking. Acta Mater. 117 (15), 321-332.

Martinez-Paneda, E., Niordson, C.F., 2016. On fracture in finite strain gradient plasticity. Int. J. Plast. 80, $154-167$.

Monchiet, V., Bonnet, G., 2013. A Gurson-type model accounting for void size effects. Int. J. Plast. 50 (2), $320-327$.

Motz, C., Weygand, D., Senger, J., Gumbsch, P., 2008. Micro-bending tests: a comparison between three-dimensional discrete dislocation dynamics simulations and experiments. Acta Mater. 56 (9), 1942-1955.

Nix, W.D., Gao, H.J., 1998. Indentation size effects in crystalline materials: a law for strain gradient plasticity. J. Mech. Phys. Solids 46 (3), $411-425$.

Ouyang, C.J., Li, Z.H., Huang, M.S., Fan, H.D., 2010. Cylindrical nano-indentation on metal film/elastic substrate system with discrete dislocation plasticity analysis: a simple model for nano-indentation size effect. Int. J. Solids Struct. 47 (22-23), 3103-3114.

Placidi, L., 2016. A variational approach for a nonlinear one-dimensional damage-elasto-plastic second-gradient continuum model. Contin. Mech. Therm. 28 (1), 119-137.

Ran, J.Q., Fu, M.W., Chan, W.L., 2013. The influence of size effect on the ductile fracture in micro-scaled plastic deformation. Int. J. Plast. 41, 65-81.

Ran, J.Q., Fu, M.W., 2014. A hybrid model for analysis of ductile fracture in micro-scaled plastic deformation of multiphase alloys. Int. J. Plast. 61, 1-16.

Ryu, I.L.L., Cai, W., Nix, W.D., Gao, H.J., 2016. Anisotropic size-dependent plasticity in face-centered cubic micropillars under torsion. JOM 68 (1), $253-260$.

Sajjadi, S.A., Ezatpour, H.R., Beygi, H., 2011. Microstructure and mechanical properties of $\mathrm{Al}_{-} \mathrm{Al}_{2} \mathrm{O}_{3}$ micro and nanocomposites fabricated by stir casting. Mater. Sci. Eng. A 528 (29-30), 8765-8771.

Sciarra, F.M.D., 2012. Hardening plasticity with nonlocal strain damage. Int. J. Plast. 34, 114-138.

Scudino, S., Liu, G., Prashanth, K.G., Bartusch, B., Surreddi, K.B., Murty, B.S., Eckert, J., 2009. Mechanical properties of Al-based metal matrix composites reinforced with Zr-based glassy particles produced by powder metallurgy. Acta Mater. 57 (6), 2029 -2039.

Siddiq, A., Schmauder, S., Huang, Y.G., 2007. Fracture of bicrystal metal/ceramic interfaces: a study via the mechanism-based strain gradient crystal plasticity theory. Int. J. Plast. 23 (4), 665-689.

Shi, Z., Huang, Y.G., Song, J., Hwang, K.C., Li, M., 2009. Study of plastic shear localization via the flow theory of mechanism-based strain gradient plasticity. ASCE J. Eng. Mech. 135 (3), 132-138.

Smyshlyaev, V.P., Fleck, N.A., 1996. The role of strain gradients in the grain size effect for polycrystals. J. Mech. Phys. Solids 44 (4), 465-495.

Solanki, K.N., Bammann, D.J., 2010. A thermodynamic framework for a gradient theory of continuum damage. Acta Mech. 213 (1), $27-38$.

Stolken, J.S., Evans, A.G., 1998. A microbend test method for measuring the plasticity length scale. Acta. Mater. 46 (14), $5109-5115$.

Tang, H., Choi, Y.S., Acharya, A., Saigal, S., 2004. Effects of lattice incompatibility induced hardening on the fracture behavior of ductile single crystals. J. Mech. Phys. Solids 52 (12), 2841-2867.

Tang, H., Acharya, A., Saigal, S., 2005. Directional dependence of crack growth along the interface of a bicrystal with symmetric tilt boundary in the presence of gradient effects. Mech. Mater. 37 (5), 593-606.

Taylor, M.B., Zbib, H.M., Khaleel, M.A., 2002. Damage and size effect during superplastic deformation. Int. J. Plast. 18 (3), $415-442$.

Voyiadjis, G.Z., Dorgan, R.J., 2001. Gradient formulation in coupled damage-plasticity. Arch. Mech. 53 (4-5), 565-597.

Voyiadjis, G.Z., Abu Al-Rub, R.K., 2005. Gradient plasticity theory with a variable length scale parameter. Int. J. Solids Struct. 42 (14), $3998-4029$.

Wen, J., Huang, Y.G., Huang, K.C., Liu, C., Li, M., 2005. The modified Gurson model accounting for the void size effect. Int. J. Plast. 21 (2), $381-395$.

Wu, B., Liang, L.H., Ma, H.S., Wei, Y.G., 2012. A trans-scale model for size effects and intergranular fracture in nanocrystalline and ultra-fine polycrystalline metals. Comput. Mater. Sci. 57, 2-7.

Xia, Z.C., Hutchinson, J.W., 1996. Crack tip fields in strain gradient plasticity. J. Mech. Phys. Solids 44 (10), 1621-1648.

Xiao, X.Z., Song, D.K., Xue, J.M., Chu, H.J., Duan, H.L., 2015. A size-dependent tensorial plasticity model for FCC single crystal with irradiation. Int. J. Plast. 65, 152-167.

Yang, Y., Yao, N., Soboyejo, W.O., Tarquinio, C., 2008. Deformation and fracture in micro-tensile tests of freestanding electrodeposited nickel thin films. Scr. Mater. 58 (12), 1062-1065.

Yi, D.K., Wang, T.C., Xiao, Z.M., 2010. Strain gradient theory based on a new framework of non-local model. Acta Mech. 212 (1), 51-67.

Yu, S.W., Feng, X.Q., 1997. Damage Mechanics. Tsinghua University Press, Beijing. 
Zbib, H.M., Aifantis, E.C., 1992. On the gradient-dependent theory of plasticity and shear banding. Acta Mech. 92 (1), $209-225$.

Zhang, X., Aifantis, K.E., Ngan, A.H.W., 2014. Interpreting the stress-strain response of Al micropillars through gradient plasticity. Mater. Sci. Eng. A 591, $38-45$.

Zhao, H.W., Zhong, Y.X., Ma, Z.C., 2016. Effects of indentation depth on micro hardness and scratch behavior of thin composite laminate. J. Alloy Compd. 680, 105-108.

Zheng, Q., Shimizu, T., Yang, M., 2016. Scale effect on springback behavior of pure titanium foils in microbending at elevated temperature. J. Mater. Process Tech. 230, 233-243.

Zhu, C.Y., Harrington, T., Livescu, V., Gray III, G.T., Vecchio, K.S., 2016. Determination of geometrically necessary dislocations in large shear strain localization in aluminum. Acta Mater. 118, 383-394. 